\title{
Evaluation of Central Auditory Processing in Musicians and Non-Musicians
}

\author{
Senanur Kahraman* \\ Sena Karaduman \\ Selim Ünsal \\ Fulya Yalçınkaya
}

\begin{abstract}
Purpose: Auditory processing is the analysis of sounds' attributions such as localization, lateralization, temporality, frequency, loudness, phase, auditory memory and auditory attention through primer and secondar central auditory paths. This study aims to show that music contributes on auditory processing by applying central auditory tests on two groups who are musicians and nonmusicians.

Materials and Methods: The participants in this study are aged between 20 and 40 . The mean age of the musician participants is $24.40 \pm 3.811$, when the mean age of non-musician participants is $26.07 \pm 4.525$. Each group has 30 participants. All participants got examined for otorhinolaryngology and they were tested for pure tone audiometry and immitansmetric measurement. For musician participants, this study included people who have professional music career for at least 5 years and for non-musician participants, it has been included those who are capable of normal hearing. All participants tested for Frequency Patterns Test (FPT), Duration Patterns Test (DPT), Random Gap Detection Test (RGDT) and Staggered Spondaic Word Test (SSWT).

Results: The results of the FPT, DPT, RGDT and SSWT tests applied to musician and non-musician participants indicate that there is a statistical significance on auditory processing between these two groups $(p<0.05)$. It is founded that there is a considerable difference as a result of statistical significance between the tests from right to left and from left to right $(p<0.05)$. In addition, it is founded that musician participants have much more comprehension ability in the tests from left to right than the tests from right to lefts.

Conclusion: This study indicates that people who are interested in music professionally have developed auditory processing skills and senses than people who does not show any professional interest in music.
\end{abstract}

Keywords: Tinnitus; hearing, central auditory processing. 


\section{INTRODUCTION}

Music activates many points in the brain and provides interaction between both hemispheres 1. The right hemisphere is responsible for the parts of music related to timbre, perception of loudness, intonation, and expression of emotions. The left hemisphere, on the other hand, processes analytical aspects of music such as rhythm speech, duration, temporal sequencing, and synchronicity ${ }^{2}$ stated that making music at a professional level is one of the most complex humanitarian abilities ${ }^{3}$. For example, a pianist should have the ability to coordinate the notes in the entire piece of music so that it can maintain integrity. Making music involves combining sensory and motor information while also paying attention to performance. That is, being able to play an instrument involves combining many different sensory and motor information at the same time and using feedback mechanisms to monitor its performance at the same time ${ }^{4}$. Tervaniemi et al. stated that the musician's brain is like an orchestra. In this orchestra, separate activities are performed individually, but the result is a holistic structure like an orchestra ${ }^{5}$. The musician should constantly control his instrument to regulate the sounds and motor movements he produces while playing 6 . Musicians begin their musical education at an early age and have to practice for hours a day throughout their careers in order to develop their special sensorymotor skills ${ }^{7}$. Central Auditory Processing (CAP); it is the auditory system responsible for functions that include sound localization, lateralization, auditory discrimination, auditory pattern awareness, temporal processing, decreased auditory performance versus incompatible or split acoustic signals. It is the analysis of the acoustic phonetic codes of the heard message in auditory ways. Central Auditory Processing Disorder (CAPD) is a difficulty manifested by poor performance in one or more of the functions listed above. Temporal processing is defined as the ability to solve and follow rapid temporal changes occurring in time. Temporal processing is very important for the ability to understand speech in noise and quiet environments ${ }^{8}$. Although it was determined in biophysical and behavioral studies that professional musicians performed better in some auditory processing functions compared to nonmusicians, musicians were able to distinguish smaller frequency differences compared to non-musicians1. The organization of sounds in music is based on their relationship to each other, and what is understood from a musical sound mostly depends on its relationship to the following or accompanying sound ${ }^{9}$. There are many tests that evaluate central auditory processing. In our study, temporal processing was evaluated using the Random Gap Detection Test (RGDT), Frequency Patterns Test (FPT) and Duration Patterns Test (DPT). In addition, the Staggered Spondaic Word Test (SSWT) was performed with dicotic stimuli. These tests are sensitive to detecting dysfunctions of major brain regions such as the brainstem, corpus callosum, right and left temporal lobe. In the light of the researches made from these tests, it is expected that better results will be obtained from those who receive music education compared to normal individuals ${ }^{10,11}$. The aim of this study is to reveal the functionality of central auditory processing of musicians and non-musicians with RGDT, FPT, DPT and SSWT tests.

\section{MATERIALS AND METHODS}

This study was conducted in Istanbul Medipol University, Faculty of Health Sciences, Department of Audiology. Approval was obtained from the Non-Invasive Clinical Research Ethics Committee of Istanbul Medipol University (03.01.2018 dated and 34 decision number) ${ }^{12}$.

Individuals: The ages of the individuals participating in this study range between 20 and 40 . The mean age of musicians is $24.40 \pm 3.811$, and the mean age of nonmusicians is $26.07 \pm 4.525$. There were 30 people in both the musician group and the non-musician group. In the musician group ${ }^{13}$ women, 17 men, and non-musicians 17 women and 13 men took part. Pure tone audiometry and immitansmetric measurements were performed on all participants after the Otorhinolaryngology measurement. Individuals who were interested in professional music for at least 5 years and had normal hearing were included in the study. In addition, 2 participants in the non-musician group and 12 participants in the musician group had tinnitus. In the musician group, the number of people who were interested in music for 5-10 years was 21 , and the number of those who were interested in more than 10 years was 9 .

Method: The participants filled in an informed consent form describing the study and the test and a pre-study form containing some information to be used in the study. In our study, RGDT, FPT, DPT and SSWT tests were performed with the sounds recorded in the computerloaded MP3 format. Philips SHP1900 model supraaural headphones were used.

Random Gap Detection Test (RGDT): Tonal stimulus pairs were used in the Random Gap Detection Test. The frequencies of tonal stimuli were 500, 1000, 2000 and $4000 \mathrm{~Hz}$. The duration of the stimulus was set to $17 \mathrm{~ms}$, the descent and rise time $1 \mathrm{~ms}$, the gaps between stimuli to $0-40 \mathrm{~ms}(0,2,5,10,15,20,25,30,40)$ and presented in a randomly prepared order 12 . The test was performed at frequencies of $500,1000,2000$ and $4000 \mathrm{~Hz}$, respectively. The participant was asked to verbally say that he heard one or two voices. Later, in whatever msec interval he started to detect the interval (if he said he heard 2 stimuli), that was determined as the threshold of the participant. Frequency Patterns Test (FPT): According to this, FPT was developed by Pinheiro and Ptacek13. Creating the CD version of the test, Musiek F. (1994) stated that two voices with frequencies of $880 \mathrm{~Hz}$ and $1122 \mathrm{~Hz}$ were used $^{14}$. Three sequential stimuli randomly arranged from two sounds were used in our study. The sounds were $200 \mathrm{~ms}$ in duration, the time between sounds was 150 $\mathrm{ms}$, and the descending and rising times were $10 \mathrm{~ms}$. Stimuli at these two frequencies have equal perception of 
intensity. The individual was asked to describe the sounds in the pattern given in terms of finesse and thickness in order of arrival. For example, when the 880-1120-1120 $\mathrm{Hz}$ stimulus was given respectively, the individual was expected to define thick-thin-thin. 50 stimuli were played to each participant. Duration Patterns Test (DPT): In this test, 2 sounds with a frequency of $1000 \mathrm{~Hz}, 500 \mathrm{~ms}$ and $250 \mathrm{~ms}$ duration were used. 3 randomly arranged stimuli were generated from these two sounds. The intervals between sounds were $300 \mathrm{msec}$. Participants were asked to describe and say the sounds in the pattern given according to their order in terms of length and brevity. For example; If 500-500-250 msec stimulus was given, respectively, the individual was expected to define longlong-short. In our study, individuals were played 50 stimuli. Before the measurement, 5 stimulus tests were used to teach and were not scored 2,15.

Staggered Spondaic Word Test (SSWT): In this test with words, compound words; it was presented in right ear (right non-competing), dichotic right ear (right competing), dichotic left ear (left competing), and left ear (left noncompeting) positions. In this test, 44 in total, 4 exercises, 40 were divided as test items. The participant was asked to repeat the words he heard when he heard them. In the first four exercises, he was warned if he was not obeying the commands or if he was making a mistake. The results were converted into percentage success based on dicotic right and left ear responses.

\section{Statistical Evaluation of Data}

Statistical data used in the study were prepared with the SPSS 25.0 (Statistical Package for Social Sciences) computer program. Kolmogorov-Smirnov test was used to determine whether the groups showed normal distribution. Mann Whitney $U$ Test and Independent $t$ test were used to compare the test batteries used in the study in two groups and to evaluate the differences. The presence of tinnitus was compared between the groups with the Chi-Square test.

\section{RESULTS}

Central auditory processing tests were applied to the participants with and without musicians, and the results obtained were compared with the Mann Whitney $U$ test. Both ears participated in the statistical analysis. The presence of tinnitus was evaluated between the groups with the Chi-Square test and a statistically significant difference was observed between the groups $(p<0,05)$.

Random Gap Detection Test (RGDT): Measurements were made at frequencies of 500,1000, 2000 and 4000 $\mathrm{Hz}$ in both groups. In the analysis, the responses of the two groups at each frequency were compared. A comparison was made between the groups, taking into account the significance values for each threshold. The results obtained from musicians and non-musicians were analyzed using the Mann Whitney $U$ Test and a statistically significant difference was observed between groups for four frequencies $(p<0.05)$. In our study, unlike other studies, it was seen that there was some long threshold, and the reasons for this will be investigated in future studies 16 . The comparison of the scores of musicians and non-musicians according to RGDT test results is shown in Table 1.

Duration Patterns Test (DPT): DPT test was applied to both groups. Test results were evaluated and scored according to their success percentage. The MannWhitney $U$ test was applied to analyze whether there was a difference between groups in the test percentages obtained, and a significant difference was found between groups as a result of the test $(p=0,046)$. The mean values of musicians are higher than the normal group formed by non-musicians. The results are shown in Table 2.

Frequency Patterns Test (FPT): The FPT test was applied to both groups and the test results were scored according to their success percentage. The right and left ears of the groups were evaluated separately. A statistically significant difference was found between the groups in the test percentages obtained $(p=0,000)$. The results are shown in Tables 3 and 4.

Staggered Spondaic Word Test (SSWT): In the SSWT test, the test results were evaluated according to the success percentages, considering the correct pronunciation of the presented words. The test results were divided into 3 categories: general percentage, right to left, left to right. Independent t test was used to analyze whether there was a difference between groups in the test percentages obtained and a significant difference was found in the general results according to the test results $(p<0.001)$. The results are shown in Table 5.

Table 1: Comparison of RGDT test in musicians and non-musicians.

\begin{tabular}{ccccc}
\hline & $\mathbf{5 0 0 ~ H z}$ & $\mathbf{1 0 0 0 ~ H z}$ & $\mathbf{2 0 0 0 ~} \mathbf{~ H z}$ & $\mathbf{4 0 0 0 ~} \mathbf{~ H z}$ \\
\hline Musicians & 6.37 & 6.83 & 6,63 & 6.43 \\
Non-Musicians & 10.1 & 11.33 & 12,6 & 11,4 \\
p value & $0.027^{*}$ & $0.036^{*}$ & $0.017^{*}$ & $0.021^{\star}$ \\
\hline
\end{tabular}

Table 2: Comparison of DPT test in musicians and non-musicians.

\begin{tabular}{ccc}
\hline Participants & DPT test mean percentage values & p value \\
\hline Musicians & $84.60 \%$ & $0.046^{\star}$ \\
Non-Musicians & $73.23 \%$ & 0. \\
\hline
\end{tabular}


Table 3: Mean values of FPT for the right ear.

\begin{tabular}{ccc}
\hline Participants & Right ear FPT test mean percentage values & P value \\
\hline Musicians & $88.83 \%$ & $0.000^{\star}$ \\
Non-Musicians & $61.97 \%$ & \\
\hline
\end{tabular}

Table 4: Mean FPT values for the left ear.

\begin{tabular}{ccc}
\hline Participants & Left ear FPT test mean percentage values & \\
\hline Musicians & $89.33 \%$ & $0.000^{\star}$ \\
Non-Musicians & $61.67 \%$ & \\
\hline
\end{tabular}

Table 5: Comparison of SSWT test in musicians and non-musicians.

\begin{tabular}{cccc}
\hline & SSWT overall mean percentages & $\begin{array}{c}\text { Mean percentages from right to } \\
\text { left }\end{array}$ & $\begin{array}{c}\text { Mean percentages from left to } \\
\text { right }\end{array}$ \\
\hline Musicians & 68,3 & 64,6 & 72 \\
Non-musicians & 50,3 & 48,6 & 51,6 \\
P value & $0.001^{*}$ & $0.001^{*}$ & $0.001^{*}$ \\
\hline
\end{tabular}

\section{DISCUSSION}

Some regions are specifically active in the processing of music in the brain. Frontal lobe and hippocampus are involved in combining auditory input by providing a connection between both hemispheres. In addition, the subcortical region and auditory cortex are the regions responsible for listening to music, corpus callosum for musical memory and music content, amygdala and cerebellar vermis for emotional reactions to music, temporal lobe language centers and frontal lobe focusing on lyrics, singing and remembering ${ }^{16}$, cerebellum for dancing, holding tempo and playing instruments. Professional musicians often engage in music for long hours during the day. This long exposure time is thought to bring along their superior auditory abilities. Music education not only enhances auditory perception, but also has an impact on linguistic and cognitive development. Consistent with perceptual improvement studies, better results have been obtained in individuals who have also received music training in electrophysiological measurements ${ }^{17,18}$. In our study, comparisons were made between groups with RGDT, DPT, FPT and SSWT tests. Musicians performed better than non-musicians in the RGDT test at $500,1000,2000$ and $4000 \mathrm{~Hz}$. The mean of detecting random gaps was lower in the musician group. Finding the means in a shorter period of time suggests that the central auditory system performs faster. Similarly, musicians were more successful than non-musicians in DPT, FPT and SSWT tests, and there were statistically significant differences between the groups. High performance in auditory functions was also observed in electrophysiological studies with musicians. In the study of to differentiate the sounds of different qualities placed in a series of continuously given sounds with the same quality, musicians were successful in the grouping type that required more complex analysis, while nonmusicians were successful only in the simpler pitch harmony grouping. This study measured the mechanisms before attention processes, and superior performance was observed in musicians in both grouping tasks ${ }^{19}$.
These results are similar to our study results. Differences between musicians and non-musicians were also detected in measurements of auditory compatibility, and musicians were relatively more successful in determining incompatible timbre ${ }^{20}$. In addition to these, a study found that musicians process the timbre of the human voice better, and accordingly, musical timbre processing has been interpreted as providing high performance in processing the timbre of other sound sources ${ }^{21}$. All components of music activate different points in the brain. Koelsch et al. (2005) to find out which points in the brain the perception of music activates with the fMRI method. As a result of this research, strong activations were observed in the frontal operculum and superior temporal gyrus of the brain17. The fMRI method showed Schneider et al. (2002) the Heschl's gyrus, Schlaug et al. (1995) the corpus callosum is activated by music in their study ${ }^{22}$ 23. The activations demonstrated objectively by fMRI were also demonstrated with central tests in our study, and the results obtained support each other. Gaser and Schlaug (2003) found differences in the motor, auditory and visual-spatial regions of the brain between professional and amateur piano players and those who were not interested in musical performance. They found a positive correlation between musician status (professionalism or amateur status) and the primary somatosensor, premotor, anterior-superior parietal regions, and gray matter volume in the bilateral inferior temporal gyrus. In other words, it has been shown that the gray matter volume increases in these regions as we progress from amateurishness to professionalism ${ }^{4}$. Rammsayer and Altenmuller (2006) studied 36 musicians and 36 non-musicians to examine different temporal processing abilities (with auditory fusion, temporal discrimination, rhythm perception tests). It was found that musicians performed better than individuals without musical experience in distinguishing temporal discrimination, rhythm perception, auditory fusion abilities and tone duration ${ }^{24}$. Similarly, temporal sequencing ability of FPT and DPT norms is measured. Nascimento et al. (2010) in their study, applied the FPT 
test to 20 violinists and 20 people who were not interested in music, and it was observed that the results were better for musicians. The result of this study supports the result of our study ${ }^{10}$. in their study comparing the DPT test results of 15 violin players, 15 vocalists and nonmusicians, they stated that musicians had significantly better central auditory processing skills. It has also been reported that vocalists have better results than musicians playing violin, and these results are consistent with the results of our study ${ }^{15}$. in their studies where musicians evaluated the auditory evoked potentials specific to instrument sounds, they could not find a statistically significant difference between the groups. They linked these results to the common experiences of musicians ${ }^{12}$. In contrast to the results obtained in our study, Majak and Śliwinska-Kowalska (2016) did not find any difference between the groups in their study in which they applied RGDT, FPT, DPT tests to a total of 36 participants, aged 19 to 24 , in their study with 18 music academy students and 18 university students and other Central Auditory Processes Tests have been conducted and similar results were found in the tests performed ${ }_{25}$. Similarly observed in their study that there was no significant difference in RGDT thresholds between musicians and non-musicians 11. In our study, RGDT, which measures the ability to detect short time intervals between two stimuli, was used to evaluate temporal resolution, which is a subcategory of temporal processing, and a statistically significant difference was found between the groups. Musicians can detect the time intervals between two stimuli in a shorter time than non-musicians. In addition, a statistically significant difference was found between the groups in the SSWT test in our study. SSWT test is a test that measures binaural combining and integration. Since there are no studies in the literature regarding this test, no comparison was made possible. In our study, a statistically significant difference was obtained between the groups. According to these results, it was concluded that binaural combining in the brainstem, corpus callosum and cortical centers was better in musicians. aspects.

\section{CONCLUSION}

Music provides the activation of many regions in the brain. With these activations, the processing, combining, timing of auditory information properties can be made. Auditory processing functions of the brain can be measured with central auditory processing tests. As a result of our study, it was revealed that musicians have better auditory processing skills with central auditory processing tests. Therefore, it is recommended that people should be interested in music, either professionally or as a hobby. However, a higher rate of tinnitus was observed among musicians compared to non-musicians. Therefore, musicians should take the necessary precautions to prevent their hearing systems from being affected.

\section{CONFLICT OF INTEREST}

The authors declares no conflict of interest

\section{ETHICS COMMITTEE APPROVAL}

The study was conducted in accordance with the principles of the Declaration of Helsink.

\section{REFERENCES}

1. Akın O, Belgin E. Hearing Characteristics and Frequency Discrimination Ability in Musicians and Nonmusicians. J Int Adv Otol. 2009;5:195-202.

2. ASHA I. Central Auditory Processing Disorders. 2008;2:1-6.

3. Münte TF, Altenmüller E, Jancke L. The musician's brain as a model of neuroplasticity. Nature Reviews Neuroscience. 2003; 3: 473-8.

4. Gaser C, Schlaug G. Brain structures differ between musicians and non-musicians. J Neurosci. 2003; 23: 9240-45.

5. Tervaniemi M. Musicians-same or different? Ann N Y Acad Sci. 2005;1169 151-56.

6. Zatorre RJ, Chen JL, Penhune VB. When brain plays music: auditory-motor interactions in music perceptionand production. Nat Rev Neurosci. 2007;8: 547-58.

7. Bangert M, Schlaug G. Specialization of the specialized in features of external human brain morphology. Eur J Neurosci. 2004; 24:1832-1834.

8. Geffner D. Central Auditory Processing Disorders-Definition, Description, and Behaviors. Auditory Processing DisordersAssesment, Management, and Treatment Plural Publishing, Inc. 2004;6:1-6.

9. Limb CJ. Structural and functional neural correlates of music perception. Anat Rec A Discov Mol Cell Evol Biol. 2006; 288:435-6.

10. Nascimento FM, Martinez Monteiro RA, Debus Soares C, da Costa Ferreira MI. Temporal Sequencing Abilities in Musicians Violinists and Non-Musicians. Int Arch Otorhinolaryngol. 2010;14: 217-224.

11. Monteiro RAM, Nascimento FM, Soares CD, Ferreira MI DC. Temporal resolution abilities in musicians and non musicians violinists. Int Arch Otorhinolaryngol. 2010;14: 302-8.

12. Shahin A, Bosnyak DJ, Trainor LJ, Roberts LE. Enhancement of neuroplastic $\mathrm{P} 2$ and rawN1c auditory evoked potentials in musicians. J Neurosci. 2003; 23:45-52.

13. Johnson M, Bellis TJ, Billiet C. Audiological Assesment of (C) APD. Processing Disorders-Assesment, Management, and Treatment (s.75-94). UK: Plural Publishing, Inc. 2005;2:1-6.

14. Musiek F. Frequency (Pitch) and Duration Pattern Tests. J Am Acad Audiol. 2006;5: 265-8.

15. Kumar PV, Rana B, Krishna R. Temporal processing in musicians and non-musicians. J Hear Science. 2014;4: 35-42.

16. Heeke P, Vermiglio AJ, Bulla E, Velappan K. The Relationship between Random Gap Detection and Hearing in Noise Test Performances. Journal of the American Academy of Audiology. 2008; 29:948-54.

17. Koelsch S, Fritz T, Schulze K, Alsop D, Schlaug G. Adults and children processing music: An fMRI study. Neurolmage. 2006;1:68-76.

18. Yalçınkaya F, Muluk NB, Ataş A, Keith RW. Random Gap Detection Test and Random Gap Detection Test-Expanded results in children with auditory neuropathy. J Int Adv Otol. 2008; 73:1558-63. 
19. Zuijen TLV, Sussman E, Winkler I, Näätänen R, Tervaniemi M. Grouping of sequential sounds-an event related potential study comparing musicians and nonmusicians. J Cogn Neurosci. 2004;16: 331-38.

20. Minati L, Rosazza C, D'Incert L, Pietrocini E, Valentini L, et al. Functional MRI/Event-related potential study of sensory consonance and dissonance in musicians and nonmusicians, NeuroReport. 2009;20:87-92.

21. Chartrand JP, Belin P. Superior voice timbre processing in musicians. Neurosci Lett. 2006;405:164-167.

22. Schlaug G, Jancke L, Huang Y, Staiger JF, Steinmetz H. In- creased corpus callosum size in musicians. Neuropsychologia. 1995; 33:1047-55.

23. Schneider P, Scherg M, Dosch HG, Specht HJ, Gutschalk A. Morphology of Heschl's gyrus reflects enhanced activation in the auditory cortex of musicians. Nat Neurosci; 5:688-94.

24. Rammsayer T, Altenmuller E. Temporal information processing in musicians and nonmusicians. Music Percept. 2004; 24: 37-47.

25. Majak J, Śliwinska-Kowalska M. Does musical training enhance auditory processing test performance in normal hearing adults? Otorynolaryngologia. 2004;15:165-72. 